\title{
Weyl-Invariant Lightlike Branes and Soldering of Black Hole Space-Times
}

\author{
E.I. Guendelman ${ }^{1, *}$, A. Kaganovich ${ }^{1, * *}$, E. Nissimov ${ }^{2, * * *}$, and S. Pacheva ${ }^{2, \dagger}$ \\ ${ }^{1}$ Department of Physics, Ben-Gurion University of the Negev, P.O.Box 653 \\ IL-84105 Beer-Sheva, Israel \\ ${ }^{2}$ Institute for Nuclear Research and Nuclear Energy, Bulgarian Academy of Sciences \\ Boul. Tsarigradsko Chausee 72, BG-1784 Sofia, Bulgaria
}

\begin{abstract}
Key words Weyl-conformal invariance, lightlike branes, black holes.
PACS 11.25.-w, 04.70.-s, 04.50.+h

We consider self-consistent coupling of the recently introduced new class of Weyl-conformally invariant lightlike branes (WILL-branes) to $D=4$ Einstein-Maxwell system plus a $D=4$ threeindex antisymmetric tensor gauge field. We find static spherically-symmetric solutions where the space-time consists of two regions with different black-hole-type geometries and different values for a dynamically generated cosmological constant, separated by the WILL-brane which "straddles" their common event horizon. Furthermore, the WILL-brane produces a potential "well" around itself acting as a trap for test particles falling towards the horizon.
\end{abstract}

Copyright line will be provided by the publisher

\section{Introduction}

Lightlike membranes are of particular interest in general relativity as they describe impulsive lightlike signals arising in various violent astrophysical events, e.g., final explosion in cataclysmic processes such as supernovae and collision of neutron stars [1]. Lightlike membranes are basic ingredients in the so called "membrane paradigm" theory [2] which appears to be a quite effective treatment of the physics of a black hole horizon.

In refs. $[3,4]$ lightlike membranes in the context of gravity and cosmology have been extensively studied from a phenomenological point of view, i.e., by introducing them without specifying the Lagrangian dynamics from which they may originate. Recently in a series of papers $[6,7]$ we have developed a new field-theoretic approach for a systematic description of the dynamics of lightlike branes starting from concise Weyl-conformally invariant actions. The latter are related to, but bear significant qualitative differences from, the standard Nambu-Goto-type $p$-brane actions ${ }^{1}$ (here $(p+1)$ is the dimension of the brane world-volume).

In the present note we discuss spherically-symmetric solutions for the coupled system of bulk $D=4$ Einstein-Maxwell plus 3-index antisymmetric tensor gauge field interacting with a WILLbrane. The latter serves as a matter and charged source for gravity and electromagnetism and, in addition, produces a space-varying dynamical cosmological constant. The above solutions describe space-times divided into two separate regions with different black hole geometries and different values of the dynamically generated cosmological constant, separated by the WILL-brane which

\footnotetext{
* E-mail: guendel@bgumail.bgu.ac.il.

** E-mail: alexk@bgumail.bgu.ac.il.

*** Corresponding author E-mail: nissimov@inrne.bas.bg, Phone: +3592 7144720 , Fax: +3592 9753619

$\dagger$ E-mail: svetlana@inrne.bas.bg.

1 In ref.[5] brane actions in terms of their pertinent extrinsic geometry have been proposed which generically describe non-lightlike branes, whereas the lightlike branes are treated as a limiting case.
} 
automatically position itself on ("straddles") their common horizon. The matching of the physical parameters of the two black hole space-time regions ("soldering") is explicitly given in terms of the free WILL-brane coupling parameters (electric surface charge density and Kalb-Rammond coupling constant). A physically intersting implication of the above solutions is the emergence of a potential "well" around the WILL-brane trapping infalling test particles towards the common horizon.

\section{Weyl-Conformally Invariant Lightlike Branes}

In refs. $[6,7]$ we proposed the following new kind of $p$-brane action (in what follows we shall concentrate on the first nontrivial case $p=2$ ) :

$$
\begin{array}{r}
S=-\int d^{3} \sigma \Phi(\varphi)\left[\frac{1}{2} \gamma^{a b} \partial_{a} X^{\mu} \partial_{b} X^{\nu} G_{\mu \nu}-\sqrt{F_{a b} F_{c d} \gamma^{a c} \gamma^{b d}}\right] \\
-q \int d^{3} \sigma \varepsilon^{a b c} \mathcal{A}_{\mu} \partial_{a} X^{\mu} F_{b c}-\frac{\beta}{3 !} \int d^{3} \sigma \varepsilon^{a b c} \partial_{a} X^{\mu} \partial_{b} X^{\nu} \partial_{c} X^{\lambda} \mathcal{A}_{\mu \nu \lambda}
\end{array}
$$

The first significant difference of (1) w.r.t. standard Nambu-Goto-type $p$-brane action is the presence of a new non-Riemannian reparametrization-covariant integration measure density: $\Phi(\varphi) \equiv$ $\frac{1}{3 !} \varepsilon_{i j k} \varepsilon^{a b c} \partial_{a} \varphi^{i} \partial_{b} \varphi^{j} \partial_{c} \varphi^{k},(a, b, c=0,1,2, i, j, k=1,2,3)$, built in terms of auxiliary worldvolume scalar fields $\varphi^{i}$. As usual $\gamma_{a b}$ denotes the intrinsic Riemannian metric on the brane worldvolume and $\gamma \equiv \operatorname{det}\left\|\gamma_{a b}\right\|$. The second important difference is the "square-root" Maxwell term ${ }^{2}$ involving an auxiliary world-volume gauge field $A_{a}$ with $F_{a b}=\partial_{a} A_{b}-\partial_{b} A_{a} . G_{\mu \nu}(\mu, \nu=0,1,2,3)$ denotes Riemannian metric on the embedding $D=4$ space-time. The second Chern-Simmons-like term in (1), describing a coupling to external $D=4$ space-time electromagnetic field $\mathcal{A}_{\mu}$, is a special case of a class of Chern-Simmons-like couplings of extended objects to external electromagnetic fields proposed in ref.[9]. The last term is a Kalb-Ramond-type coupling to external space-time rank 3 gauge potential $\mathcal{A}_{\mu \nu \lambda}$.

The action (1) is manifestly invariant under Weyl (conformal) symmetry: $\gamma_{a b} \longrightarrow \gamma_{a b}^{\prime}=\rho \gamma_{a b}$, $\varphi^{i} \longrightarrow \varphi^{i}=\varphi^{\prime i}(\varphi)$ with Jacobian $\operatorname{det}\left\|\frac{\partial \varphi^{\prime}}{\partial \varphi^{j}}\right\|=\rho$.

Let us recall the physical significance of $\mathcal{A}_{\mu \nu \lambda}$ [10]. In $D=4$ when adding kinetic term for $\mathcal{A}_{\mu \nu \lambda}$ coupled to gravity (see Eq.(5) below), its field-strength $\mathcal{F}_{\kappa \lambda \mu \nu}=4 \partial_{[\kappa} \mathcal{A}_{\lambda \mu \nu]}=\mathcal{F} \sqrt{-G} \varepsilon_{\kappa \lambda \mu \nu}$ with a single independent component $\mathcal{F}$ produces dynamical (positive) cosmological constant $K=$ $\frac{4}{3} \pi G_{N} \mathcal{F}^{2}$.

Invariance under world-volume reparametrizations allows to introduce the standard (synchronous) gauge-fixing conditions: $\gamma^{0 i}=0(i=1,2), \gamma^{00}=-1$. With the latter gauge choice and using the short-hand notation $\left(\partial_{a} X \partial_{b} X\right) \equiv \partial_{a} X^{\mu} G_{\mu \nu} \partial_{b} X^{\nu}$, the equations of motion for the brane action (1) read:

$$
\left(\partial_{0} X \partial_{0} X\right)=0 \quad, \quad\left(\partial_{0} X \partial_{i} X\right)=0 \quad, \quad\left(\partial_{i} X \partial_{j} X\right)-\frac{1}{2} \gamma_{i j} \gamma^{k l}\left(\partial_{k} X \partial_{l} X\right)=0,
$$

these are in fact constraints analogous to the (classical) Virasoro constraints of string theory;

$$
\partial_{i} X^{\mu} \partial_{j} X^{\nu} \mathcal{F}_{\mu \nu}(\mathcal{A})=0 \quad, \quad \partial_{i} \chi+\sqrt{2} q \partial_{0} X^{\mu} \partial_{i} X^{\nu} \mathcal{F}_{\mu \nu}(\mathcal{A})=0
$$

(here $\chi \equiv \frac{\Phi(\varphi)}{\sqrt{-\gamma}}$ plays the role of variable brane tension, $\mathcal{F}_{\mu \nu}(\mathcal{A})=\partial_{\mu} \mathcal{A}_{\nu}-\partial_{\nu} \mathcal{A}_{\mu}$ );

$$
\begin{array}{r}
\widetilde{\square}^{(3)} X^{\mu}+\left(-\partial_{0} X^{\nu} \partial_{0} X^{\lambda}+\gamma^{k l} \partial_{k} X^{\nu} \partial_{l} X^{\lambda}\right) \Gamma_{\nu \lambda}^{\mu} \\
-q \frac{\gamma^{k l}\left(\partial_{k} X \partial_{l} X\right)}{\sqrt{2} \chi} \partial_{0} X^{\nu} \mathcal{F}_{\lambda \nu} G^{\lambda \mu}-\frac{\beta}{3 !} \varepsilon^{a b c} \partial_{a} X^{\kappa} \partial_{b} X^{\lambda} \partial_{c} X^{\nu} G^{\mu \rho} \mathcal{F}_{\rho \kappa \lambda \nu}=0,
\end{array}
$$

2 "Square-root" Maxwell (Yang-Mills) action in $D=4$ was originally introduced in the first ref.[8] and later generalized to "square-root" actions of higher-rank antisymmetric tensor gauge fields in $D \geq 4$ in the second and third refs.[8]. 
where $\mathcal{F}_{\rho \kappa \lambda \nu}=4 \partial_{[\kappa} \mathcal{A}_{\lambda \mu \nu]}$ as above, $\widetilde{\square}^{(3)} \equiv-\frac{1}{\chi \sqrt{\gamma^{(2)}}} \partial_{0}\left(\chi \sqrt{\gamma^{(2)}} \partial_{0}\right)+\frac{1}{\chi \sqrt{\gamma^{(2)}}} \partial_{i}\left(\chi \sqrt{\gamma^{(2)}} \gamma^{i j} \partial_{j}\right)$, where $\gamma^{(2)} \equiv \operatorname{det}\left\|\gamma_{i j}\right\|(i, j=1,2)$, and $\Gamma_{\nu \lambda}^{\mu}=\frac{1}{2} G^{\mu \kappa}\left(\partial_{\nu} G_{\kappa \lambda}+\partial_{\lambda} G_{\kappa \nu}-\partial_{\kappa} G_{\nu \lambda}\right)$ is the affine connection corresponding to the external space-time metric $G_{\mu \nu}$.

The first Virasoro-like constraint in (2) explicitly exhibits the inherent lightlike property of the brane model (1), hence the acronym WILL (Weyl-invariant light-like) brane.

\section{Bulk Gravity-Matter Coupled to WILL-brane}

Let us now consider the coupled Einstein-Maxwell-WILL-brane system adding also a coupling to a rank 3 gauge potential:

$$
S=\int d^{4} x \sqrt{-G}\left[\frac{R(G)}{16 \pi G_{N}}-\frac{1}{4} \mathcal{F}_{\mu \nu} \mathcal{F}^{\mu \nu}-\frac{1}{4 ! 2} \mathcal{F}_{\kappa \lambda \mu \nu} \mathcal{F}^{\kappa \lambda \mu \nu}\right]+S_{\text {WILL-brane }}
$$

Here $\mathcal{F}_{\mu \nu}=\partial_{\mu} \mathcal{A}_{\nu}-\partial_{\nu} \mathcal{A}_{\mu}, \mathcal{F}_{\kappa \lambda \mu \nu}=4 \partial_{[\kappa} \mathcal{A}_{\lambda \mu \nu]}=\mathcal{F} \sqrt{-G} \varepsilon_{\kappa \lambda \mu \nu}$ as above, and the WILL-brane action is the same as in (1).

The equations of motion for the WILL-brane subsystem are the same as (2)-(4), whereas the equations for the space-time fields read:

$$
\begin{array}{r}
R_{\mu \nu}-\frac{1}{2} G_{\mu \nu} R=8 \pi G_{N}\left(T_{\mu \nu}^{(E M)}+T_{\mu \nu}^{(\text {rank-3) }}+T_{\mu \nu}^{(\text {brane })}\right), \\
\partial_{\nu}\left(\sqrt{-G} G^{\mu \kappa} G^{\nu \lambda} \mathcal{F}_{\kappa \lambda}\right)+q \int d^{3} \sigma \delta^{(4)}(x-X(\sigma)) \varepsilon^{a b c} F_{b c} \partial_{a} X^{\mu}=0, \\
\varepsilon^{\lambda \mu \nu \kappa} \partial_{\kappa} \mathcal{F}+\beta \int d^{3} \sigma \delta^{(4)}(x-X(\sigma)) \varepsilon^{a b c} \partial_{a} X^{\lambda} \partial_{a} X^{\mu} \partial_{a} X^{\nu}=0 .
\end{array}
$$

The energy-momentum tensors read: $T_{\mu \nu}^{(E M)}=\mathcal{F}_{\mu \kappa} \mathcal{F}_{\nu \lambda} G^{\kappa \lambda}-G_{\mu \nu} \frac{1}{4} \mathcal{F}_{\rho \kappa} \mathcal{F}_{\sigma \lambda} G^{\rho \sigma} G^{\kappa \lambda}$,

$$
\begin{aligned}
& T_{\mu \nu}^{(\text {rank-3) }}=\frac{1}{3 !}\left[\mathcal{F}_{\mu \kappa \lambda \rho} \mathcal{F}_{\nu}{ }^{\kappa \lambda \rho}-\frac{1}{8} G_{\mu \nu} \mathcal{F}_{\kappa \lambda \rho \sigma} \mathcal{F}^{\kappa \lambda \rho \sigma}\right]=-\frac{1}{2} \mathcal{F}^{2} G_{\mu \nu} \\
& T_{\mu \nu}^{(\text {brane })}=-G_{\mu \kappa} G_{\nu \lambda} \int d^{3} \sigma \frac{\delta^{(4)}(x-X(\sigma))}{\sqrt{-G}} \chi \sqrt{-\gamma} \gamma^{a b} \partial_{a} X^{\kappa} \partial_{b} X^{\lambda} .
\end{aligned}
$$

For the bulk gravity-matter system coupled to a charged WILL-brane (5) we find the following static spherically symmetric solutions. The bulk space-time consists of two regions separated by the WILL-brane sitting on ("straddling") a common horizon of the former:

$$
(d s)^{2}=-A_{(\mp)}(r)(d t)^{2}+\frac{1}{A_{(\mp)}(r)}(d r)^{2}+r^{2}\left[(d \theta)^{2}+\sin ^{2}(\theta)(d \phi)^{2}\right],
$$

where the subscript $(-)$ refers to the region inside, whereas the subscript $(+)$ refers to the region outside the horizon at $r=r_{0} \equiv r_{\text {horizon }}$ with $A_{(\mp)}\left(r_{0}\right)=0$. The interior region is a Schwarzschildde-Sitter space-time:

$$
A(r) \equiv A_{(-)}(r)=1-K_{(-)} r^{2}-\frac{2 G_{N} M_{(-)}}{r}, \quad \text { for } r<r_{0},
$$

whereas the exterior region is Reissner-Norström-de-Sitter space-time:

$$
A(r) \equiv A_{(+)}(r)=1-K_{(+)} r^{2}-\frac{2 G_{N} M_{(+)}}{r}+\frac{G_{N} Q^{2}}{r^{2}}, \quad \text { for } r>r_{0},
$$

with Reissner-Norström (squared) charge given by $Q^{2}=8 \pi q^{2} r_{0}^{4}$. The rank 3 tensor gauge potential together with its Kalb-Rammond-type coupling to the WILL-brane produce via Eq.(8) a 
dynamical space-varying cosmological constant which is different inside and outside the horizon: $K_{( \pm)}=\frac{4}{3} \pi G_{N} \mathcal{F}_{( \pm)}^{2}$ for $r \geq r_{0} \quad\left(r \leq r_{0}\right), \mathcal{F}_{(+)}=\mathcal{F}_{(-)}-\beta$. The Einstein Eqs. (6) and the $X^{\mu_{-}}$ brane Eqs.(4) yield two matching conditions for the normal derivatives w.r.t. the horizon of the space-time metric components:

$$
\left.\left(\partial_{r} A_{(+)}-\partial_{r} A_{(-)}\right)\right|_{r=r_{0}}=-16 \pi G_{N} \chi,\left.\left(\partial_{r} A_{(+)}-\partial_{r} A_{(-)}\right)\right|_{r=r_{0}}=-\frac{\left.r_{0}\left(2 q^{2}+\beta^{2}\right) \partial_{r} A_{(-)}\right|_{r=r_{0}}}{2 \chi+\beta r_{0} \mathcal{F}_{(-)}} .
$$

The latter conditions allow to express all physical parameters of the solution, i.e., two spherically symmetric black hole space-time regions "soldered" along a common horizon via the WILL-brane in terms of 3 free parameters $(q, \beta, \mathcal{F})$ where (cf. Eq.(1)): (a) $q$ is the WILL-brane surface electric charge density; (b) $\beta$ is the WILL-brane (Kalb-Rammond-type) charge w.r.t. rank 3 space-time gauge potential $\mathcal{A}_{\lambda \mu \nu} ;$ (c) $\mathcal{F}_{(-)}$is the vacuum expectation value of the 4 -index field-strength $\mathcal{F}_{\kappa \lambda \mu \nu}$ in the interior region. For the common horizon radius, the Schwarzschild and Reissner-Nordström masses we obtain:

$$
\begin{array}{r}
r_{0}^{2}=\frac{1}{4 \pi G_{N}\left(\mathcal{F}_{(-)}^{2}-\beta \mathcal{F}_{(-)}+q^{2}+\frac{\beta^{2}}{2}\right)} \quad, \quad M_{(-)}=\frac{r_{0}\left(\frac{2}{3} \mathcal{F}_{(-)}^{2}-\beta \mathcal{F}_{(-)}+q^{2}+\frac{\beta^{2}}{2}\right)}{2 G_{N}\left(\mathcal{F}_{(-)}^{2}-\beta \mathcal{F}_{(-)}+q^{2}+\frac{\beta^{2}}{2}\right)} \\
M_{(+)}=M_{(-)}+\frac{r_{0}}{2 G_{N}\left(\mathcal{F}_{(-)}^{2}-\beta \mathcal{F}_{(-)}+q^{2}+\frac{\beta^{2}}{2}\right)}\left(2 q^{2}+\frac{2}{3} \beta \mathcal{F}_{(-)}-\frac{1}{3} \beta^{2}\right) .
\end{array}
$$

For the brane tension we get accordingly: $\chi=\frac{r_{0}}{2}\left(q^{2}+\frac{\beta^{2}}{2}-2 \beta \mathcal{F}_{(-)}\right)$.

Using expressions (14)-(15) we find for the slopes of the metric coefficients $A_{( \pm)}(r)$ at $r=r_{0}$ :

$$
\left.\partial_{r} A_{(+)}\right|_{r=r_{0}}=-\left.\partial_{r} A_{(-)}\right|_{r=r_{0}},\left.\quad \partial_{r} A_{(-)}\right|_{r=r_{0}}=8 \pi G_{N} \chi=4 \pi G_{N} r_{0}\left(q^{2}+\frac{\beta^{2}}{2}-2 \beta \mathcal{F}_{(-)}\right) \text {. }
$$

In view of (16) (and assuming for definiteness $\beta>0$ ) we conclude:

(i) In the area of parameter space $\mathcal{F}_{(-)}>\frac{q^{2}+\frac{\beta^{2}}{2}}{2 \beta}$ (i.e., when $\chi<0$ - negative brane tension) the common horizon is: (a) the de-Sitter horizon from the point of view of the interior Schwarzschildde-Sitter geometry; (b) it is the external Reissner-Nordström horizon (the larger one) from the point of view of the exterior Reissner-Nordström-de-Sitter geometry.

(ii) In the opposite area of parameter space $\mathcal{F}_{(-)}<\frac{q^{2}+\frac{\beta^{2}}{2}}{2 \beta}$ (i.e., when $\chi>0$ - positive brane tension) the common horizon is: (a) the Schwarzschild horizon from the point of view of the interior Schwarzschild-de-Sitter geometry; (b) it is the internal (the smaller one) Reissner-Nordström horizon from the point of view of the exterior Reissner-Nordström-de-Sitter geometry.

Now let us consider planar motion of a (charged) test patricle with mass $m$ and electric charge $q_{0}$ in a gravitational background given by the solutions in Section 3. Conservation of energy yields $\frac{E^{2}}{m^{2}}={r^{\prime}}^{2}+V_{e f f}^{2}(r)(E, J$ - energy and orbital momentum of the test particle; prime indicates proper-time derivative) with:

$$
\begin{array}{cc}
V_{e f f}^{2}(r)=A_{(-)}(r)\left(1+\frac{J^{2}}{m^{2} r^{2}}\right)+\frac{2 E q_{0}}{m^{2}} \sqrt{2} q r_{0}-\frac{q_{0}^{2}}{m^{2}} 2 q^{2} r_{0}^{2} & \left(r \leq r_{0}\right) \\
V_{e f f}^{2}(r)=A_{(+)}(r)\left(1+\frac{J^{2}}{m^{2} r^{2}}\right)+\frac{2 E q_{0}}{m^{2}} \frac{\sqrt{2} q r_{0}^{2}}{r}-\frac{q_{0}^{2}}{m^{2}} \frac{2 q^{2} r_{0}^{4}}{r^{2}} \quad\left(r \geq r_{0}\right)
\end{array}
$$

where $A_{(\mp)}$ are the same as in (12) and (13). Taking into account (16) we see that in the parameter interval $\mathcal{F}_{(-)} \in\left(\frac{q^{2}+\frac{\beta^{2}}{2}}{\beta}, \infty\right)$ the (squared) effective potential $V_{e f f}^{2}(r)$ acquires a potential "well" in the vicinity of the WILL-brane (the common horizon) with a minimum on the brane itself. 
In the simplest physically interesting case with $q=0, \mathcal{F}_{(-)}=\beta$ and $\beta$ - arbitrary, i.e., matching of Schwarzschild-de-Sitter interior (with dynamically generated cosmological constant) against pure Schwarzschild exterior (with no cosmological constant) along the WILL-brane as their common horizon, the typical form of $V_{e f f}^{2}(r)$ is graphically depicted in Fig.1.

Thus, we conclude that if a test particle moving towards the common event horizon loses energy (e.g., by radiation), it may fall and be trapped by the potential well, so that it neither falls into the black hole nor can escape back to infinity and, as a result, a "cloud" of trapped particles is formed around the WILL-brane materialized horizon.

\section{Acknowledgements.}

E.N. and S.P. are supported by European RTN network "Constituents, Fundamental Forces and Symmetries of the Universe" (contract No.MRTN-CT-2004-005104). They also received partial support from Bulgarian NSF grant F-1412/04. Finally, all of us acknowledge support of our collaboration through the exchange agreement between the Ben-Gurion University of the Negev (Beer-Sheva, Israel) and the Bulgarian Academy of Sciences.

\section{References}

[1] C. Barrabés and P. Hogan, "Singular Null-Hypersurfaces in General Relativity", World Scientific, Singapore (2004).

[2] K. Thorne, R. Price and D. Macdonald (eds.), "Black Holes: The Membrane Paradigm", Yale Univ. Press, New Haven, CT (1986).

[3] W. Israel, Nuovo Cim. B44, 1 (1966); erratum, ibid B48, 463 (1967).

[4] C. Barrabés and W. Israel, Phys. Rev. D43, 1129 (1991); T. Dray and G. 't Hooft, Class. Quantum Grav. 3, 825 (1986).

[5] C. Barrabés and W. Israel, Phys. Rev. D71, 064008 (2005) (gr-qc/0502108).

[6] E. Guendelman, A. Kaganovich, E. Nissimov and S. Pacheva, hep-th/0409078; in "Second Workshop on Gravity, Astrophysics and Strings", edited by P. Fiziev et.al. (Sofia Univ. Press, Sofia, Bulgaria, 2005) (hep-th/0409208); in "Third Internat. School on Modern Math. Physics", Zlatibor (Serbia and Montenegro), edited by B. Dragovich and B. Sazdovich (Belgrade Inst. Phys. Press, 2005) (hepth/0501220).

[7] E. Guendelman, A. Kaganovich, E. Nissimov and S. Pacheva, Phys. Rev. D72, 0806011 (2005) (hepth/0507193); hep-th/0611022.

[8] H.B. Nielsen and P. Olesen, Nucl. Phys. B57, 367 (1973); A. Aurilia, A. Smailagic and E. Spallucci, Phys. Rev. D47, 2536 (1993) (hep-th/9301019); A. Aurilia and E. Spallucci, Class. Quantum Grav. 10, 1217 (1993).

[9] A. Davidson and E. Guendelman, Phys. Lett. 251B, 250 (1990).

[10] A. Aurilia, H. Nicolai and P. Townsend, Nucl. Phys. B176, 509 (1980); A. Aurilia, Y. Takahashi and P. Townsend, Phys. Lett. 95B, 265 (1980)

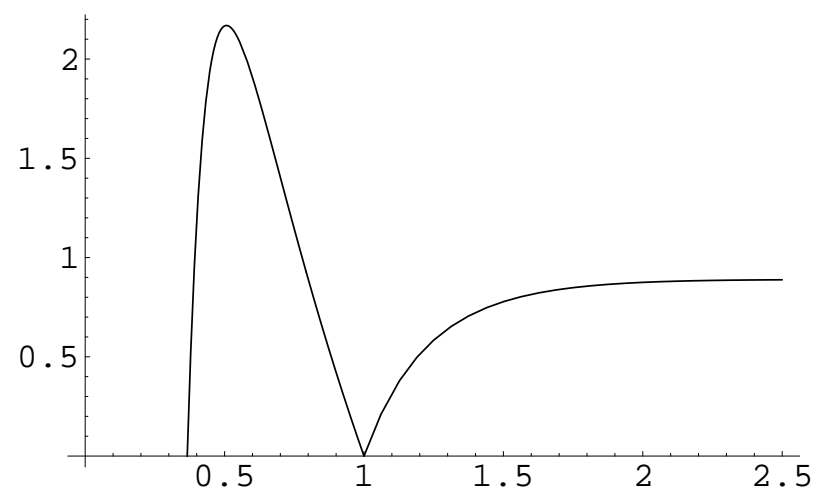

Fig. 1 Shape of $V_{e f f}^{2}(r)$ as a function of the dimensionless ratio $x \equiv r / r_{0}$ 


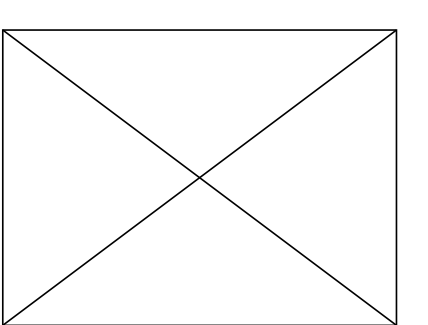

